Springer Tracts in Civil Engineering

Cecilia Bolognesi

Daniele Villa Editors

From Building

Information

Modelling

to Mixed Reality

Springer 


\section{Springer Tracts in Civil Engineering}

\section{Series Editors}

Giovanni Solari, Wind Engineering and Structural Dynamics Research Group, University of Genoa, Genova, Italy

Sheng-Hong Chen, School of Water Resources and Hydropower Engineering, Wuhan University, Wuhan, China

Marco di Prisco, Politecnico di Milano, Milano, Italy

Ioannis Vayas, Institute of Steel Structures, National Technical University of Athens, Athens, Greece 
Springer Tracts in Civil Engineering (STCE) publishes the latest developments in Civil Engineering - quickly, informally and in top quality. The series scope includes monographs, professional books, graduate textbooks and edited volumes, as well as outstanding $\mathrm{PhD}$ theses. Its goal is to cover all the main branches of civil engineering, both theoretical and applied, including:

- Construction and Structural Mechanics

- Building Materials

- Concrete, Steel and Timber Structures

- Geotechnical Engineering

- Earthquake Engineering

- Coastal Engineering; Ocean and Offshore Engineering

- Hydraulics, Hydrology and Water Resources Engineering

- Environmental Engineering and Sustainability

- Structural Health and Monitoring

- Surveying and Geographical Information Systems

- Heating, Ventilation and Air Conditioning (HVAC)

- Transportation and Traffic

- Risk Analysis

- Safety and Security

\section{Indexed by Scopus}

To submit a proposal or request further information, please contact:

Pierpaolo Riva at Pierpaolo.Riva@springer.com (Europe and Americas) Mengchu Huang at mengchu.huang@ springer.com (China)

More information about this series at http://www.springer.com/series/15088 
Cecilia Bolognesi · Daniele Villa Editors

\section{From Building Information Modelling to Mixed Reality}

第 Springer 


\section{Editors}

\author{
Cecilia Bolognesi \\ Dipartimento ABC \\ Politecnico di Milano \\ Milan, Italy
}

\author{
Daniele Villa \\ Dipartimento di Architettura \\ e Studi Urbani (DASTU) \\ Politecnico di Milano \\ Milan, Italy
}

ISSN 2366-259X

ISSN 2366-2603 (electronic)

Springer Tracts in Civil Engineering

ISBN 978-3-030-49277-9

ISBN 978-3-030-49278-6 (eBook)

https://doi.org/10.1007/978-3-030-49278-6

(C) The Editor(s) (if applicable) and The Author(s), under exclusive license to Springer Nature

Switzerland AG 2021

This work is subject to copyright. All rights are solely and exclusively licensed by the Publisher, whether the whole or part of the material is concerned, specifically the rights of translation, reprinting, reuse of illustrations, recitation, broadcasting, reproduction on microfilms or in any other physical way, and transmission or information storage and retrieval, electronic adaptation, computer software, or by similar or dissimilar methodology now known or hereafter developed.

The use of general descriptive names, registered names, trademarks, service marks, etc. in this publication does not imply, even in the absence of a specific statement, that such names are exempt from the relevant protective laws and regulations and therefore free for general use.

The publisher, the authors and the editors are safe to assume that the advice and information in this book are believed to be true and accurate at the date of publication. Neither the publisher nor the authors or the editors give a warranty, express or implied, with respect to the material contained herein or for any errors or omissions that may have been made. The publisher remains neutral with regard to jurisdictional claims in published maps and institutional affiliations.

This Springer imprint is published by the registered company Springer Nature Switzerland AG

The registered company address is: Gewerbestrasse 11, 6330 Cham, Switzerland 


\title{
From Survey to Parametric Models: HBIM Systems for Enrichment of Cultural Heritage Management
}

\author{
Sandro Parrinello and Anna Dell'Amico
}

\begin{abstract}
The paper summarizes some research experiences conducted by the DAda-LAB laboratory, of the Department of Civil Engineering and Architecture of the University of Pavia, on the theme of the digitization of large monumental complexes and, in particular, on the development of databases and 3D models for Cultural Heritage management. The connection between the digital survey and HBIM models is addressed by evaluating the most appropriate strategies for the production of parametric models by analyzing the methodologies aimed at the transformation of data from continuous systems to discrete models. From the digital acquisition phases to the development of complex information systems, drawing becomes the tool that allows to decompose and recompose the complexity of reality, organizing databases that offer opportunities for the development of management systems. The models in this way make it possible to explicate information according to specific parameters for the benefit of a more effective representation for the knowledge, management, and documentation of the Cultural Heritage.
\end{abstract}

Keywords HBIM $\cdot 3 \mathrm{D}$ model $\cdot$ Survey $\cdot$ Database $\cdot$ Heritage

\section{The Representation in Digital Models ${ }^{1}$}

The theme of the reproducibility of the heritage of historical cities keeps the debate open on the problems of structuring and sharing a typology of language made of signs and symbols that can be codified and recognized through the construction of generalizable formal schemes.

Through the drawing discipline, the construction of a graphic language is developed in which the complexity of reality is reduced in favor of the communication

\footnotetext{
S. Parrinello · A. Dell'Amico ( $\varangle)$

DICAr, University of Pavia, Pavia, Italy

e-mail: anna.dellamico@unipv.it

S. Parrinello

e-mail: sandro.parrinello@unipv.it
}

(C) The Editor(s) (if applicable) and The Author(s), under exclusive license to Springer 


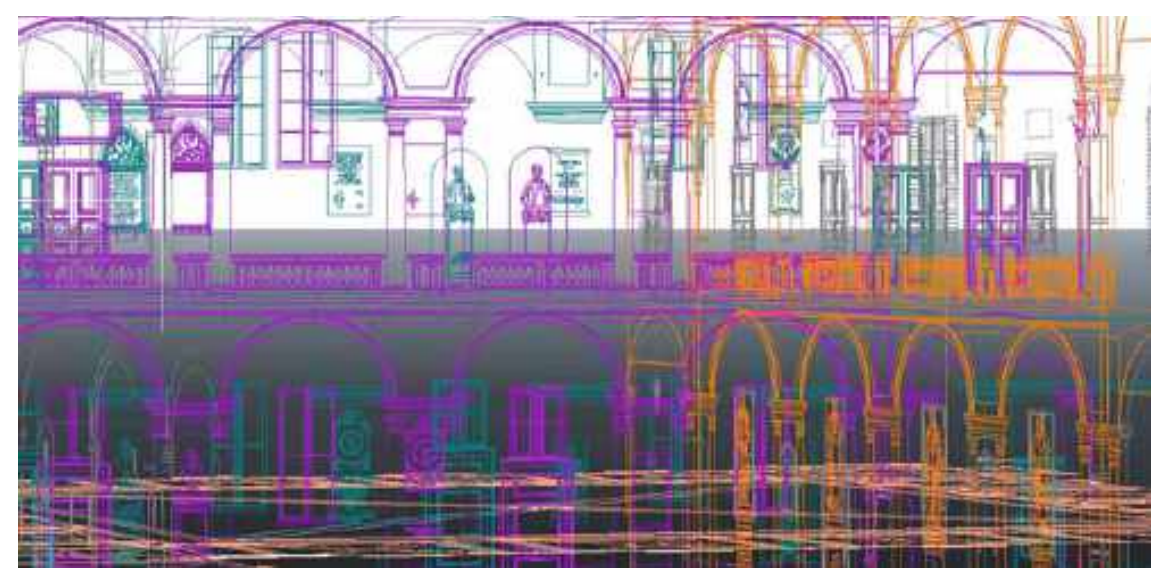

Fig. 1 Three-dimensional modeling environments: the import phase of two-dimensional files in a three-dimensional environment

of selective information. The drawing makes explicit those descriptive needs that animate the communication style. Those representative tensions focus the gesture of representing in its being a method of communication and finally, the transmission of information (Fig. 1).

The narrative system to which a specific drawing or model refers to is, therefore, part of a language which, in its compositional grammar, can be interpreted based on the concept of drawing from and for, evaluating the reality that one wants to represent and the purposes of the representation of it, through a reflection, which in some cases is implicit, also based on metric data acquisition methodologies (Cervellini 2016).

The Information can be transferred instantly anywhere and have the ability to reach anyone provided with a network connection; physical space is no longer considered enough and needs its digital extension in which objects are enriched with finite information that can become something else. The information transposed from real space into virtual space creates new digital and physical configurations that actively influence each other (Ratti 2017).

Cities, places where information are concentrated, are represented through their digital alter ego, which is nothing more than a shareable spatial expansion on which a greater number of apps and technologies are developed, that go to reproduce information and services based on different data characterization criteria (user/use). In this image extension that moves a media reformulation of the identity of the places and, more generally, a reformulation of the identity of the representation, the documentation of the historical heritage deals with the theme of the digital reproducibility of spaces through their conversion into communication platforms.

If we consider each city, its places and monuments as unique and unreproducible works, the problem of identifying the references through formal schemes is proposed, through the use of generalizable and repeatable graphic symbols of reference on 
which to be able to outline representation guidelines that can reproduce and communicate different types of information (Colarossi and Lange 1996): from those aimed at the visualization of the object to those of in-depth analysis of a descriptive technical nature relating to the metric data, parameters and attributes that allow digital models to be organized and interrogated based on the attributes that define them (Osello 2015).

If the models discover their function of simplification of reality, it is in the immersive fruition and in the ways of interacting with the digital data, in the interoperability and in the possibility of adapting the information to the different communication strategies that the image extension takes shape.

In the renewal of the informative message, the model once again faces the tensions that connote its formal identity, modifying the morphological metric reliability and the qualities that increase its verisimilitude and correspondence to reality, as a function of parameters more characterizing other aspects not strictly connected to the shape. If on the one hand the tendency is to make the digital model correspond as closely as possible to the real object, the synthesis of complexity is oriented on criteria of approximation of form and limitation of imperfection in favor of standardization of the formal buildings components and easier computation and interaction with the model element.

They are the criteria of use and communication that define the ways of constructing the shape to find in the graphics and therefore in the definition of a graphic language of the model lemma useful to make it easier to understand concepts that can also be complex.

In the history of infographics, an example of how a simplified type of language can be effective is given by Oliver Byrne ${ }^{1}$ as early as 1847 in the six books "The elements of Euclid in color" having to deal with mathematical theorems through the use of a more simplified graphic language. Which is not only a simplification of the complexity of the analytical language but also become an innovative form of reinterpretation of that same complexity which allows reforming other aspects as well.

Through the definition of a graphic language, our architectural model represents and re-presents itself, as in the "The Temple of time", a map drawn at the beginning of the nineteenth century by Emma Wilard, ${ }^{2}$ as a system that contains quantified and sized information, made discrete, limited in some way through graphic language (Fig. 2).

The design of a model implies the reading of a complexity that is filtered by an idea, an idealization of the real that is designed and therefore interpreted according to a semantic reading to become part of a form that is identified into the parametric model (Fig. 3). 
BOOK IY, PEOR RIIL PKOK.

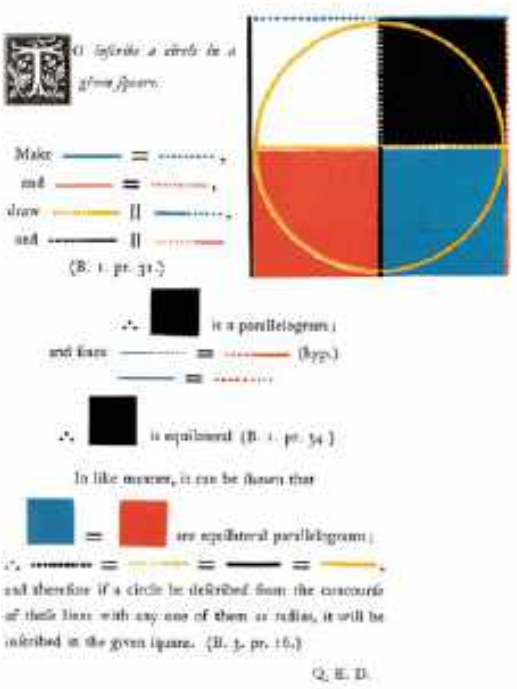

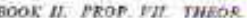

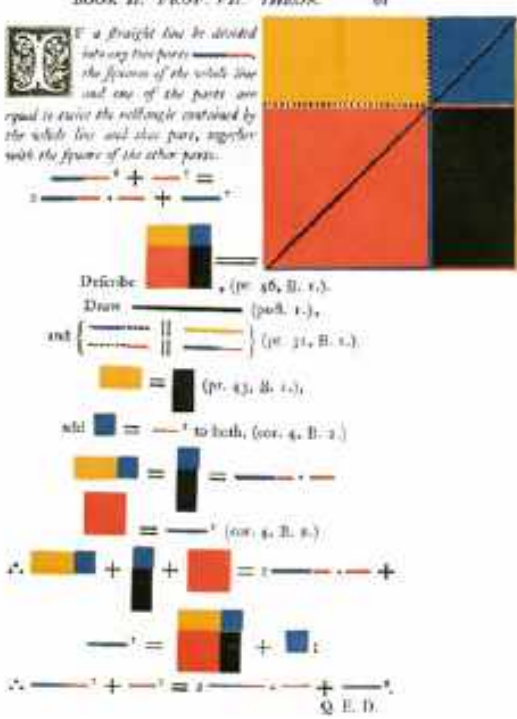

Fig. 2 Some pages taken from one of Oliver Byrne's books "The elements of Euclid in color"


Fig. 3 The infographic map "The temple of time”, Emma Willard, early nineteenth century

\section{The Discretization of the Continuum ${ }^{1}$}

The information, qualified by a different nature in terms of content and specificity of the data, finds meaning through the use of specific languages, codes in which the data take a specific and contextualized value. This context guarantees a possibility of classification based on criteria and descriptors which contribute to building indexed abacus. 
In the semantic reading of a built architectural continuum, this abacus can match, in a first simplified form of the information problem, to families of architectural elements, that are abacus proper to the model. These categories of elements require ordering attributes and qualities, also going to make explicit otherwise unexpressed particularities to accumulate the informative value of the model by adding to the more geometric-spatial qualities that use its shape. That condition of analysis of shape using a decomposition in space and the character that C. Norberg-Schulz describes well in his Genius Loci and that seemed to have found so much confirmation in the production of documented strategies based on the elaboration of digital information maps (Bertocci and Parrinello 2007), here seems to renew its sense in the development of parametric databases that are not only associated with a geometric component but that are an integral part, an inseparable whole in which the reading of the structure of the place through levels or components finds its correspondence in a universe of models organized by classes and families (Fig. 4).

In this modulation of the model shape and the redevelopment of the graphic structure of the architectural drawing, a tension is revived that concerns the reliability of the drawing and the need to guarantee that quality standard that in recent decades, through digital technologies, has characterized the research for the survey and the architectural representation.

In this perspective, reality-based models are drawn starting from the data acquired through the architectural survey and it becomes useful to evaluate which iteration systems involve the method of construction of the model to define the most appropriate methodological protocols to guarantee reliability.

It is useful to remember that a parametric model does not have as its main purpose the physical description of the asset, but intends to represent a much more complex

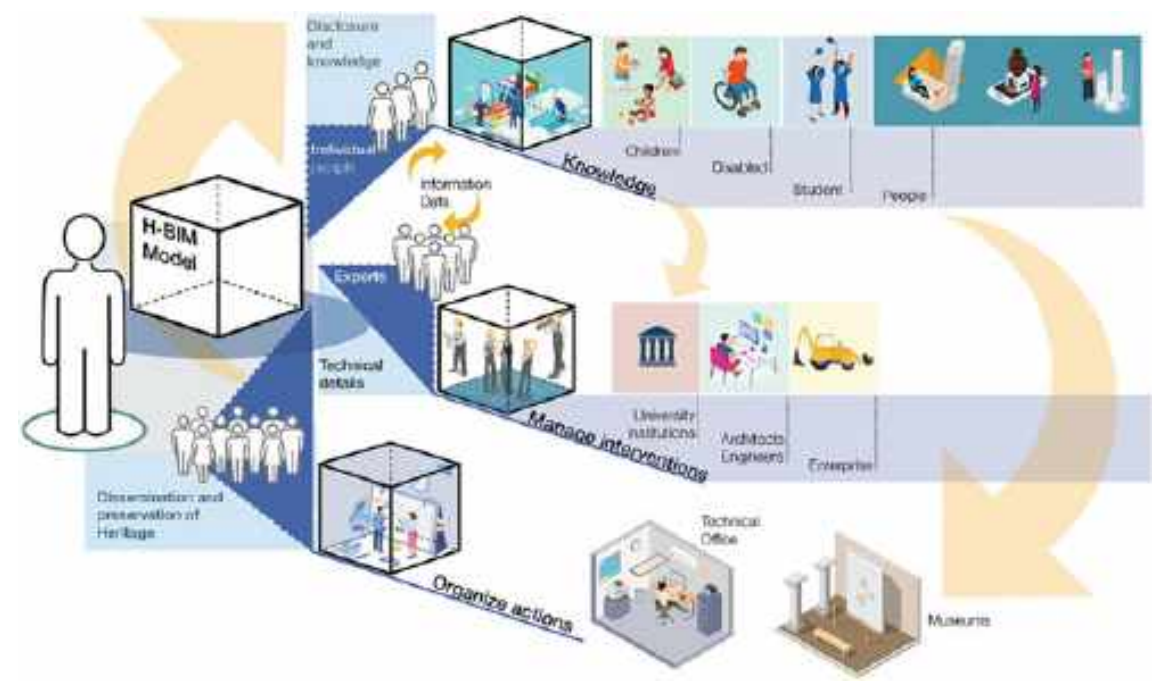

Fig. 4 Diagram of the figures involved in the management processes of the H-BIM model 


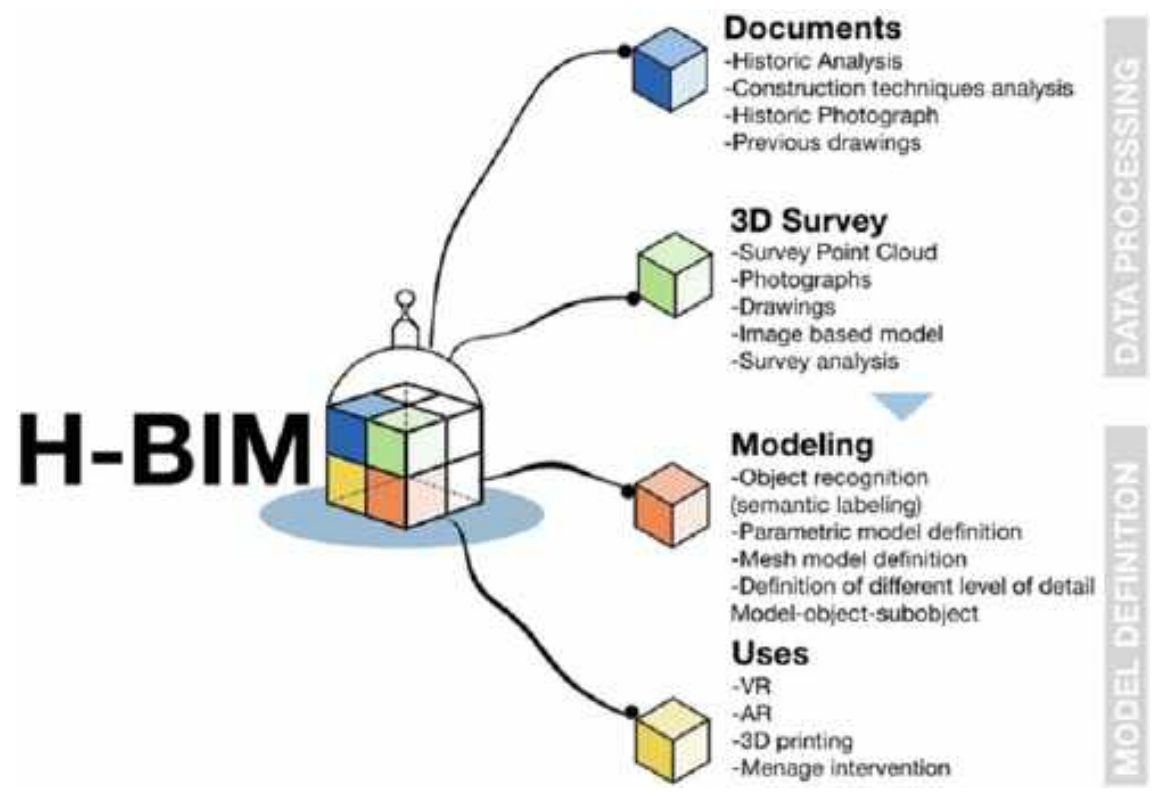

Fig. 5 Diagram of the elements that define the connotations of the three-dimensional model

system by configuring itself as a sharing information tool. The model is the shared tool that allows more people to work and cooperate, collaborating through the setting of a common language used through the same platform that finds its image in the three-dimensional model (Osello and Ugliotti 2017).

The morphometric data, connected and inseparable from its structure, qualifies instruments where different actors collaborate concerning the purposes of the process. Within a single model, which can be configured as an HBIM system, data useful for the enhancement, use, management, and control are inserted. The parametric model, of a technical nature, thus becomes a shared tool that also performs functions that are not purely technical, of the didactic, exhibition and promotional activity of cultural systems (Fig. 5).

\section{Drawing and Parametric Model ${ }^{2}$}

The software that gives the possibility to model and link information elements to the models for the management of buildings based on the BIM concept, although limited in some functions since they are specifically built for the ex-Novo design of buildings, do not exclude the possibility of construction, through a thoughtful project of information model modeling aimed at documenting the built heritage. 
The evolution of the static model (NURBS) into a parametric dynamic model benefits from the advantages of information management guaranteed by the BIM protocol.

The BIM application to the Heritage sphere implies an action of documentation of material and immaterial elements of historical structures and environments. The examples provided by the literature for cataloging and collecting data for the translation of these into an information model include buildings belonging to categories of highly protected buildings with more complete intervention projects and careful management of the life cycle.

Research on these topic by Murphy and Dore proposed and analyzed six elements of the HBIM process: heritage documentation standards, data collection techniques, $3 \mathrm{D}$ modeling concepts, as-built BIM and procedural modeling (Jordan-Palomar et al. 2018).

Of particular importance is the study on the development of standards for the sector conducted by Carleton Immersive Media development of standards for its use in traditional applications (Dore and Murphy 2017).

Speaking about cultural heritage, it is necessary to define a methodological protocol different from the approach used for modeling the design of buildings. The modeling actions require a definition of the data acquisition phases and the levels of knowledge and in-depth analysis to define a correct modeling procedure. H-BIM is focused on a protocol aimed at translating the information of the metric data, as resulting from the laser scanner survey operations, into an archetype model. The model geometries can be the result of the data editing by the comparison with the software family libraries, modifying its attributes where possible and necessary until a geometric synthesis of the forms is achieved or through in-place modeling operation of the individual objects drawn ad hoc for the different case studies ( $\mathrm{Li}$ et al. 2019).

At the basis of the modeling processes, it is good to declare the purposes of the model that will be built.

The first operation necessary for the definition of the model is the decomposition of the architectural continuum based on the analysis and cataloging of the elements to identify the appropriate modeling strategies for each of them. The elements are identified according to the first distinction between structural and decorative elements. Among these, after having defined the different categories of the model, the replicable elements that are those categories belonging to the model for which once the basic parameters have been defined are identified: it is then possible to repeat them within the model. These types of elements will be constructed through the structuring of a specific project family. For those types of elements that cannot be replicated within the model, the choice of an in-place modeling for the representation of the data as a generic model is more effective.

Once the different elements have been analyzed, and an indexed abacus of the objects that will make up the model is structured, it is possible to proceed with setting the project file. 


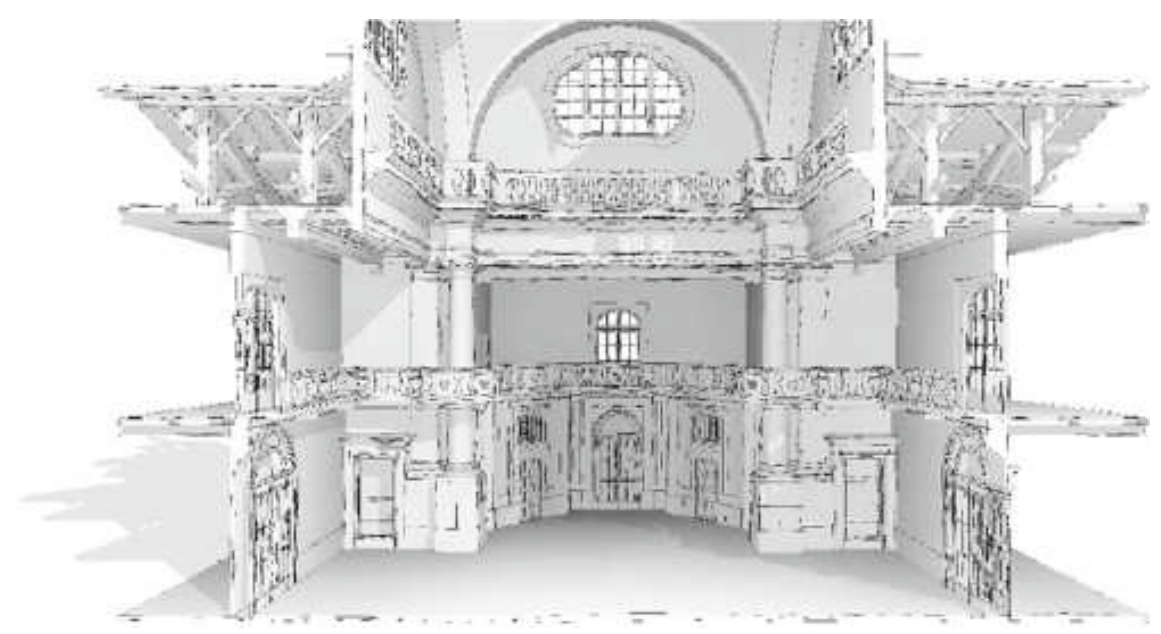

Fig. 6 An example of a HBIM model, in the cross section you can appreciate the complexity and diversification of the elements present in the model

The traditional protocol provides for the import of two-dimensional files (.dwg), like floor plans and sections, as useful traces for setting the reference levels on which to then anchor the models of the different element type families.

However, if we are working with existing buildings this requires the obligatory step, an additional phase that foresees the operations of drawing the data of the digital survey in a $2 \mathrm{D}$ environment. This time-consuming phase is not always effective for continuous monitoring of the reliability of the metric data modeled in comparison with the acquired data (Fig. 6).

The use of the two-dimensional drawing limits the geometric information to the data already processed which brings with it a first evaluation and interpretation of the real data which will inevitably add to the second interpretation of the data by those who will model and then draw for the second time the same data in three dimensions. The paradox and the question, that arises spontaneously, is: why, if the acquisition from laser scanner technology provides a three-dimensional type of data, for the elaboration of the models a passage must be then made to drawing in two dimensions and then return during the phases of the elaboration of the model into a three-dimensional environment? For this reason, methodological protocols are developed for the reduction of modeling times trying to cancel, as much as possible, the two-dimensional drawing phase of the elements. Through specific plugins ${ }^{3}$, it is possible to view the point cloud data within the software Revit. The use of plugins for viewing the point cloud has the advantage of reducing the waiting times due to the possible import, export and conversion of the scan file data into a point cloud format compatible with Revit, found that the default software prefers dialogue with the proprietary program Autodesk ReCap. This type of visualization is useful for not overloading the file in terms of bytes (Figs. 7, 8 and 9). 

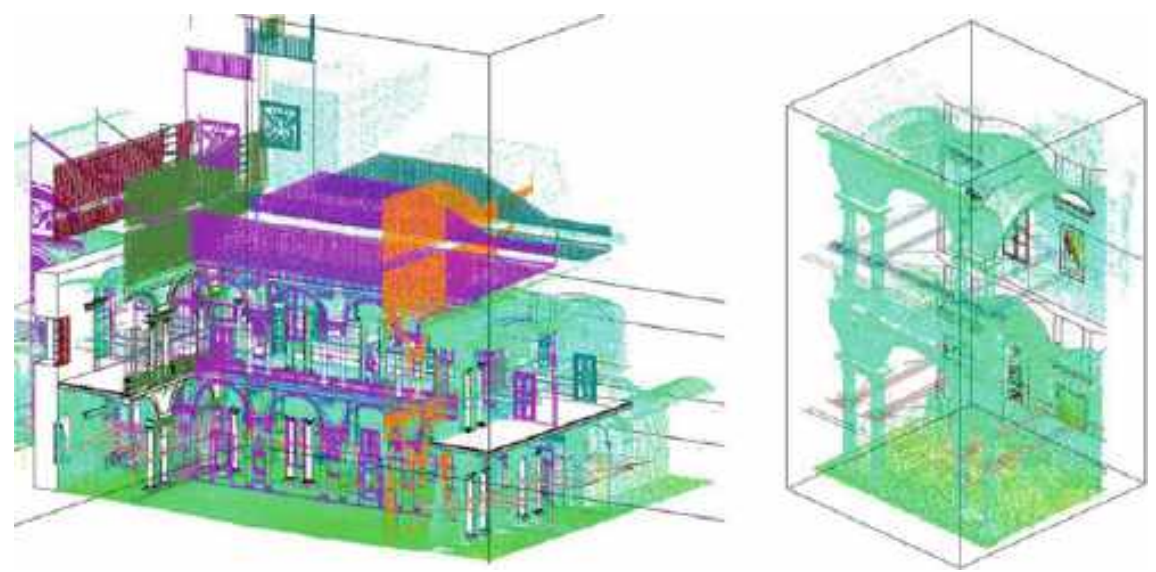

Fig. 7 Comparison between two-dimensional drawings dwg, point cloud data and 3D model imported in the same modeling environment

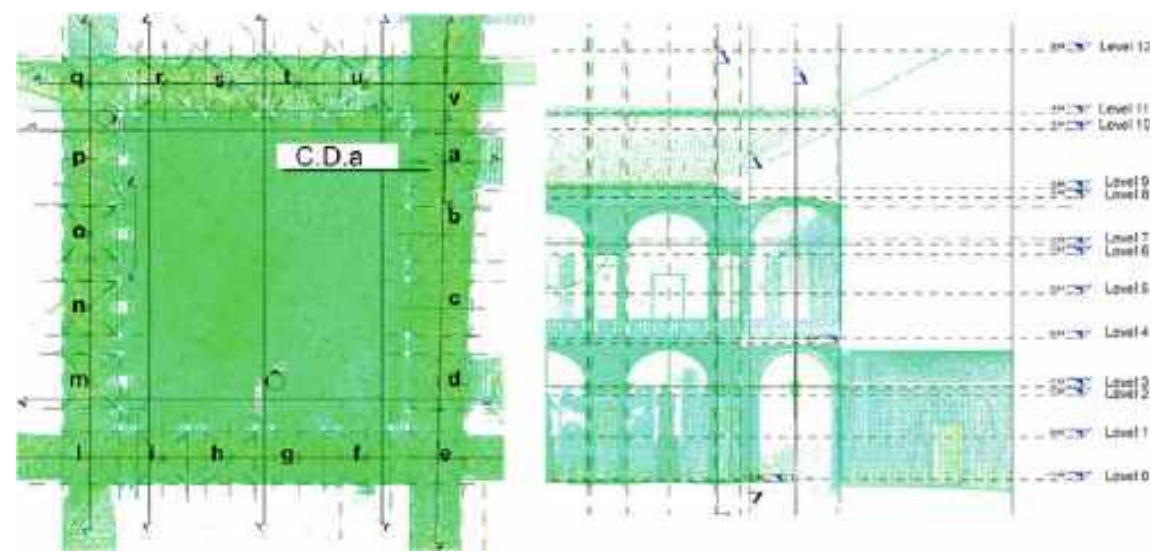

Fig. 8 The setting of the reference planes based on the point cloud data

Once the point cloud has been visualized through CloudWorx, it is possible to set the different reference levels that go to structure an ordered and indexed grid which will be the skeleton of the model, useful during the subsequent modeling phases. This display method offers the advantage of having continuous control over the correspondence and adherence between the three-dimensional data of the metric measurement and the 3D drawing of the elements.

For elements such as walls, floors, roofs, stairs, it is often possible to adapt families pre-set in the software by changing their dimensional and stratigraphic information by editing the model parameters and directly modifying the drawing lines through control points. For elements with more complex geometries such as vaults, columns, windows, balustrades or decorative elements such as statues, plaques, frames, it is 


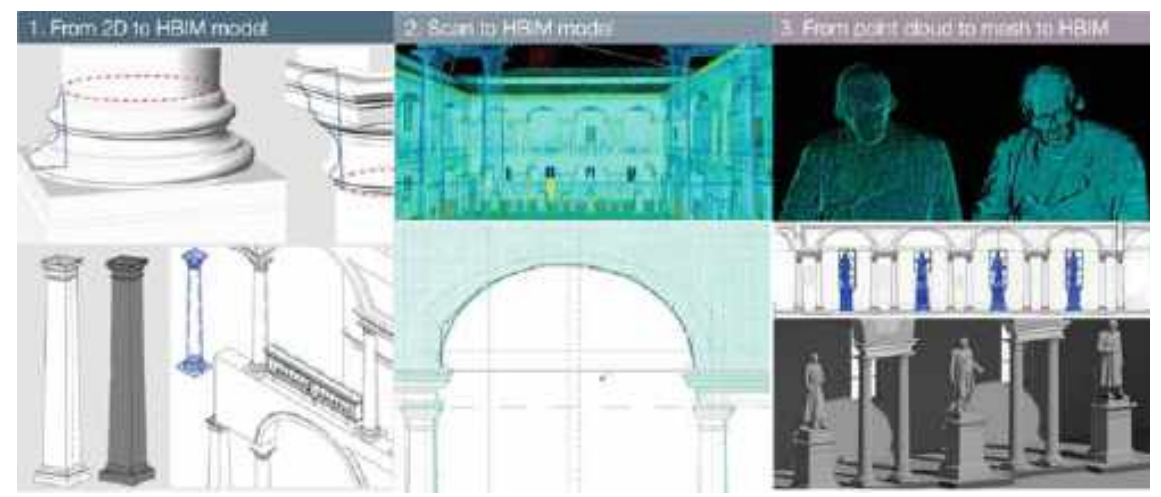

Fig. 9 The three different modeling approaches: using 2D CAD drawings, using the morphometric information directly using point cloud 3D databases, importing complex geometries after the point cloud 3D meshing

necessary to envisage a different model strategy. For these types of elements, it is recommended to evaluate whether to create a family for the element to be included in the model or whether to model it directly in the project environment as a generic model, then associating it with a specific category of elements. The example of the modeling experimentation conducted on the courtyards of the headquarters of the Central University of Pavia is here reported. ${ }^{4}$

The modeling phases of the experimentation can be divided on the basis of the three different approaches used for the representation of the different geometric complexities of the object of study. The first one based on the import of the point cloud and the Scan to BIM modeling through the creation of the model elements using families of systems, a second one dedicated to the enrichment of the descriptive detail of the model through the loading of families of elements modeled ex novo on the basis of two-dimensional drawings, and finally a third phase of mesh modeling and import of the complex geometries of the statues present in the museum.

In the first modeling phase it was possible define through the plug-in connection the basic structure of the model by defining walls, roofs, floors, stairs using families of systems present by default within the modeling program (Fig. 10).

These systems have been adapted and modified in their basic parameters through a direct control of the design of their geometry trying, by setting control points, to match the geometry of the model as much as possible to the morphometrics data given by the point cloud (Morandotti et al. 2019).

Once the basic wrapping of the model "box" was defined, the individual elements were modeled.

For those components for which it was not possible to use the predefined models of the elements, we opted for the creation of specific model families, for those elements that appear to be replicable within the environment, dividing them neatly according to their typological characters (windows, columns, doors, balustrades) or through 


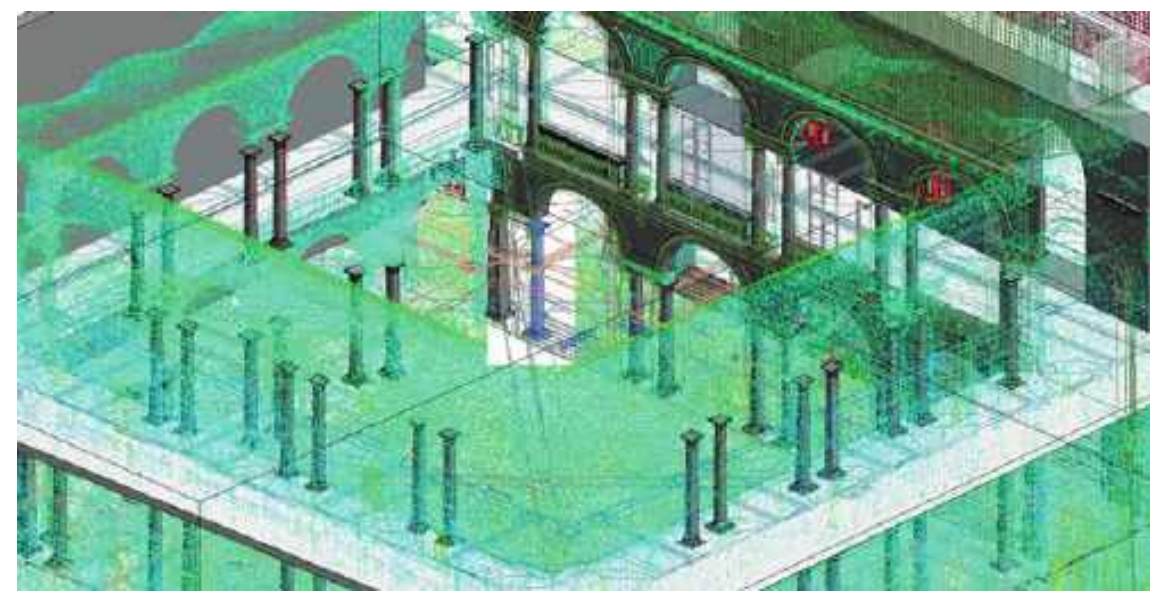

Fig. 10 View of the three-dimensional model of the courtyard of the statues (Unipv) during construction

the creation of a generic geometric model drawn in-place using the point cloud data as a guide.

In the modeling phases of the two vaulted open gallery, we can find the application of the different modeling methodologies. Given the irregularity of the elements also due to the different inclinations of the wall facings which, if observed in the planimetry, are not parallel to the wall of the colonnade, for the three-dimensional representation it was necessary to build a model escamotage by identifying a geometric solid, in this case an ellipsoid, which was as close as possible to the profile of the section in key of the vault and then go to subtract from it the excess solid material through subtraction solids configured on the reference geometric construction lines

In this case, given the presence of a double order of twin columns, the individual columns were analyzed: the data found were all different from each other imperfections due to labor and wear and tear dictated by the passage of time. For this reason, the representation of the element by type was preferred without going into the modeling detail of the individual column models with their own differences. Three model families have been created for this purpose, divided according to the macro types of column identified during the census of the elements (Morandotti et al. 2019).

For the representation of the model families, given that the software does not allow the same possibility of viewing the point cloud in the family editor, it was necessary to import the $2 \mathrm{D}$ profile drawing of the column elements, the result of the processing of the acquired data by laser scanner through which it was possible to set the different categories of model, whose construction took place through extrusion and revolution operations for the generation of model solids.

The third modeling methodology was used for the representation of the elements with complex lines and geometries such as the statues and elements present inside 

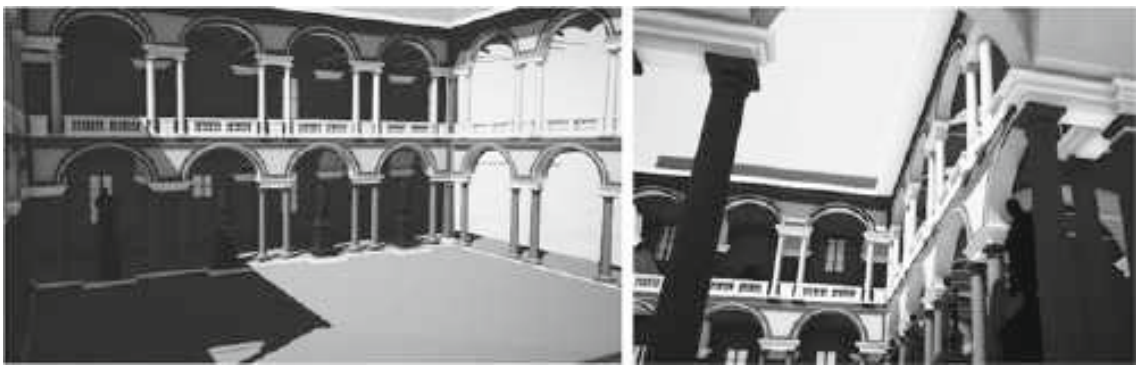

Fig. 11 3D model views

the courtyard. It was preferred to represent them using the import of mesh models generated by the processing through specific processing software. Mesh surfaces of the point cloud (Geomagic DX and MeshLab). The omission of these elements, which characterize the environment, giving it a specific identity and recognisability between the different rooms of the building would have deprived the model of essential narrative information related to the place. Once converted with a file extension compatible with the software (.dxf) these mesh models are recognized by Revit as static models and therefore cannot be modified, it is however possible to create a specific family for each element that allows you to attach specific information to the model purely descriptive character through the structuring of model parameters (Fig. 11).

\section{From General to Particular, the Model as an Information Container $^{2}$}

The research promoted in the DAda-LAB experimental laboratory of the University of Pavia, deals with the development of parametric models, as containers of information, which can interface and be suitable for the different levels of analysis and technical studies of the data. The definition of a hierarchy of virtual environments understood as containers of specific information useful for the management and development of heritage and territory management implies the possibility of defining analysis models that can be developed using the criteria of architectural representation and that can be implemented with the contribution of numerous researchers.

If on the one hand the quality and quantity of information that can be collected on the architectural object has increased and is increasing with technological development, on the other this trend entails a heterogeneity of the formats used for the documentation of the heritage, thus generating an accumulation of files with different data extensions that complicate communication and exchange between databases and professionals (Calvano 2019). New storage needs and different protocols for data management are required. The structuring of a platform as a large digital container 
that can collect different types of information, even if not necessarily compatible with each other, is still a utopian project and leads to continuous collisions with limitations problems in terms of the specific weight of the files. In the common imagination when we think of the digital world, thought associates it directly with an undefined space, limitless, with infinite possibilities; in the real context of daily use these limits still exist and during the operational phases we come across different problems due to data overload that often makes the platforms not very fluid and unmanageable or to machines in the possession of the typical user who are unable to compute a large amount of data.

The experiences aimed at managing and disseminating information relating to cultural heritage through information systems are numerous and continue their digital evolution. To be effective and useful for the management and dissemination of information, it is necessary to design the information model, in which not only are the essential guidelines for the construction of the information container model outlined but where the same information must be managed based on the diversity of contents. The information must be conveyed in a coordinated way for the management of the various contents and purposes of the operators involved in the conservation, protection, restoration, and dissemination of the heritage, who besides being users of the model become its main developers (Pavan et al. 2017).

To be able to manage information, so that it can be accessible, a management system coordinated with the tools and systems used during the life cycle of the model asset is required. Therefore, the main characteristics that a system must have are interoperability, accessibility, standardization (Agustín and Quintilla 2019).

The use of digital models for the documentation of historical heritage requires, therefore, in addition to the definition of modeling processes, also the drafting of a data sharing protocol that orders the methods of information exchange between the various disciplines and technologies. Currently, the web is the place that offers the possibility of sharing and accessing the content.

The representation of the model through different levels of detail (LoD), allows to provide a fluid visualization and efficient data access. The information modeling systems can integrate various types of information and documentation, as well as offering to different users the possibility of interconnection with the web, they allow you to actively participate during the model structuring phases (Lo Turco 2015). The involvement of various active figures in the enrichment of information requires the need for structuring between the different users a shared methodological protocol document such as a manual created specifically for the individual project that is useful to define the guidelines for the implementation of the model.

The conservation and management of the built heritage is a complex process that requires analysis at different scales, a multilevel that arises from the collaboration between different specialists in the sector.

The ordinary information that can be read by the HBIM model can include (Pocobelli et al. 2018):

- Geometric data, from the metric survey;

- Definition of schedules on architectural elements; 
- Definition of building materials. Which can be expressed graphically by the use of crosshatch, textures or by simple annotation labels;

- Surface degradations that can be described through the use of UNI glossaries and graphically expressed through the use of crosshatch and labels;

- Construction phases.

To these are added a series of metadata that can be duly linked to the model to deepen the information level:

- Archive photos;

- Texts/archival research documents;

- Data on environmental analyzes;

- Cost data;

- External links that allow connection with secondary models for the deepening of geometric data or use links to websites and different databases on the cloud (Pocobelli et al. 2018).

A type of modeling according to the definition of different information levels through the orderly organization of the elements in a digital guise has the intention of extending the life of historic buildings by shaping a new reality of our historicalcultural memory into a digital memory aimed at creating new accessible spaces (Parrinello 2019) (Fig. 12).
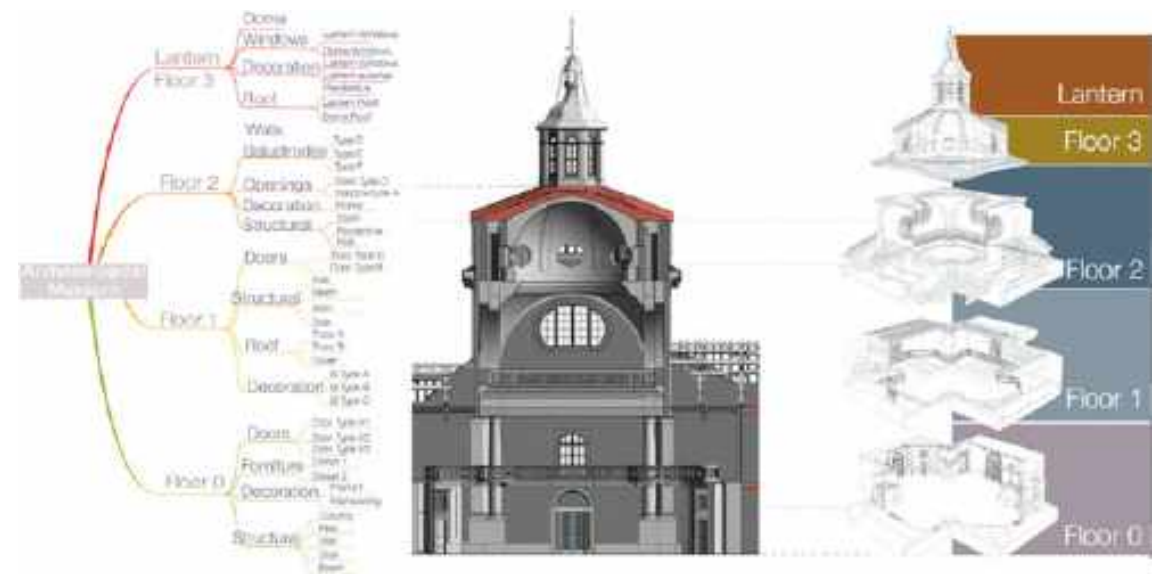

Fig. 12 The breakdown of the elements that characterize the H-BIM model, based on the category type 


\section{From Particular to General Through the Use of Virtual Environments ${ }^{2}$}

Museums and historical monuments are adapting their visit program to the ongoing social transformation. By observing the behavior of society, their content and the type of cultural offer available to citizens are changing, implementing real data with the necessary digital extension. Around the H-BIM digital model, a series of possibilities orbit according to the different fruition systems that can range from didactic, exhibition, dissemination, promotional and management activities. The programs that allow the visualization and multimedia navigation of BIM models and contents are in continuous development and updating. Two different VR visualization platforms available on the market at the time of the Autodesk Live and Twin Motion BIM experiment were tested within the research conducted. Both platforms support the use of models from PC-desktop and through the use of VR viewers (Oculus Rift). The Twin Motion platform is a free plugin that allows the direct connection between Revit and the Twin Motion editor (Unreal Engine rendering engine) with maximum compatibility and interoperability between elements. It is possible to insert the model within different scenarios, changing the environmental and lighting conditions of the scene. The model is displayed complete with the materials set on the Revit platform, however, the plugin gives the possibility to change the materials also directly using the presets set by the software material library. Lacks the possibility of deepening the information of the data, the objects are selectable and are listed by categories but it is not possible to view any additional information. The Revit Live platform works as an Autodesk cloud service. The model is sent by the Revit Live software plugin link to the cloud platform which processes the conversion of the model and sends it back to the user in a format ready for sharing the scenario with the extension.lvmd. The application is more limited in the construction of the scenario, compared to the previous one, not due to the possibility of changing the background of the landscape and the materials but maintains the characterization of the scene and material settle down on Revit. Precisely because of the compatibility guaranteed by the Autodesk manufacturer, in this case it is possible to view the information and parameters of the individual objects through information sheets structured by default and not editable. During viewing, it is possible to measure the distances from the position within the user's virtual environment with three-dimensional objects. Unlike the Twin Motion plugin, the Live is a service that Autodesk doesn't offers for a fee, the trial version limits the use of 10 uploads in 30 days. Being a sector that of virtualization and compatibility between Revit and fruition platforms, everything is still in the development phase, plug-ins and developers change quickly with system updates and present new opportunities. The same Revit Live used for this experimentation in a few months will be obsolete will no longer support the updated versions of the Revit software already the 2020 version does not have this option in its interface, because it has been replaced by new plug-in possibilities such as Unity Reflects born from the collaboration between Unity and Revit release scheduled for December 2019. This 
fact indicates that the technology is under development and the need for management protocols to be continuously monitored and kept under control also based on the possibilities provided by the different developers, adapting in some way at the time of development of management software (Figs. 13 and 14).

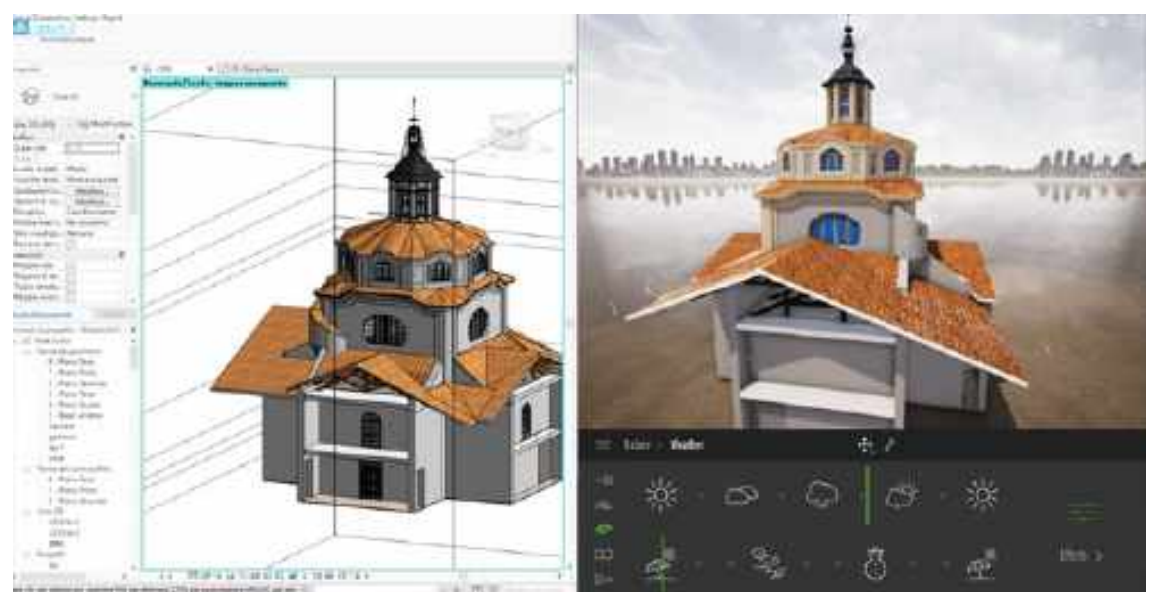

Fig. 13 The connection of the model to the twinmotion viewing platform

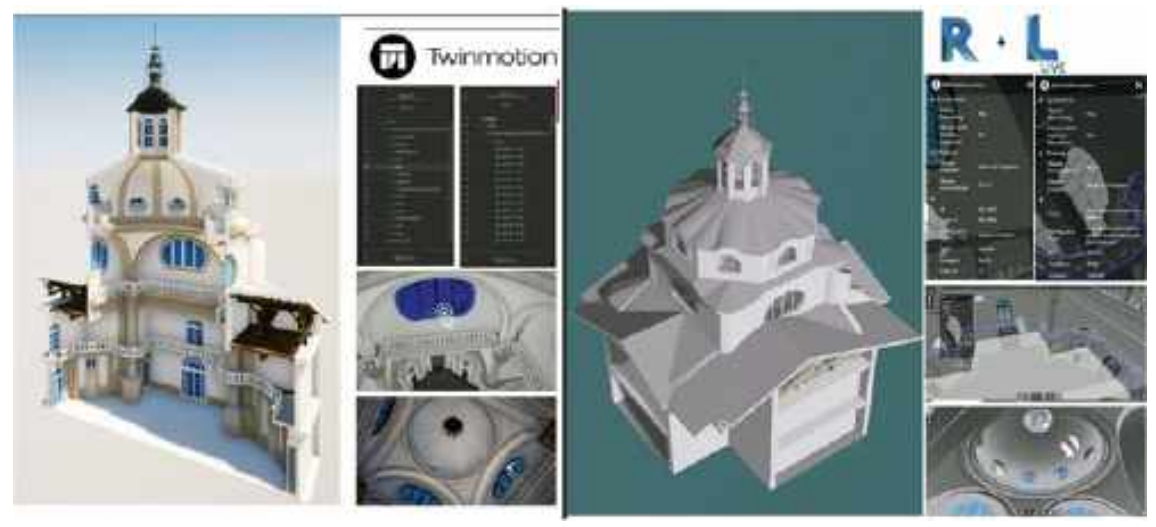

Fig. 14 Comparison between the two plugins (twinmotion and revit live) for the interactive visualization of the H-BIM model 


\section{Conclusions ${ }^{1}$}

Synthesizing reality through the development of a model implies defining in turn models based on a process of logical abstraction of forms. The digital transposition of heritage, for sharing and communication with the global society, through communication channels based on open access and social access and sharing systems, continuously searches for new possibilities of configurations and data visualization.

Advanced digital models can produce a digitalization action of global architecture, frame objects in spatial modules, structure the analyzed characteristics and transform the output of the tools into optimized 3D databases, responding to the dynamic and standard requirements of European regulations.

The data collected on site during survey activities, through the different tools, explain an overabundance of information which, if not processed and downloaded, loses their communicative function. The set of data and information, in order to become a communication tool for different users, must find the right means of explanation. Drawings, symbols, geometries, images, sounds and multimedia elements have their peculiarities and equal information dignity if justified by a specific purpose (Parrinello et al. 2019).

This is because from the analysis of the general data to the in-depth analysis, an attempt is made to understand how the parametric model is, on the one hand, interfaced with the database, on the other, it can become an enhancement tool to encourage the use of multimedia content. The model generated within the HBIM management platform can be exported and used in secondary fruition platforms for various purposes.

Speaking of historical heritage through the computer literacy of the diffused heritage it is possible to redirect tourist flows, giving visibility to those monuments or to those itineraries that are less known and frequented by the people masses, as well as allowing access at any time through the making available of a widespread digital museum, which does not want to be a substitute for the real visit experience, but rather a tool for deepening available to the visitor-widespread (Maggi 2007). Faced with an ever-changing panorama in which the tools of representation and use of the elements vary and are diversified, research is directed towards a process of the evaluation process on the potential of the various HBIM scenarios for the enhancement of historic environments. Following the directives of the new building and territorial management regulations of international policies such as the funding programs allocated by the European Commission $\mathrm{H} 2020$ and the various regulations that national and international policies are adopting, the research aims at defining through experimentation on different case studies of protocols for the management of the historical heritage built through HBIM modeling. Today, the use of diversified and unrelated protocols between the various players in the sector are generating a high quantity of digital products which, however, are disconnected from each other, generating a dispersion of information as well as generating ineffective working practices that hinder interoperability also causing economic losses, the goal of the future development is to enclose in a single modeling protocol different possibilities of use and 
programming through the use of collaborative systems that allow a better sharing of information in heritage construction projects.

\section{Notes}

${ }^{1}$ Oliver Byrne (c. 1810-c. 1880) was a writer and civil engineer of Irish origin. In addition to a treatise on euclidean geometry, the book is a new didactic expedient in which Byrne uses graphic symbols and primary colors to express more complex concepts. The author states that this is a didactic device that distinguishes its edition from all the others: "Euclid's Elements in which symbols and colored schemes replace the letters, to the benefit of those who approach this subject".

${ }^{2}$ Emma Willard (1787-1870) American activist in 1814 founded the first high school reserved for female study.

${ }^{3}$ There are different possibilities of dialogue between the point cloud and the Revit software depending on the software used to manage the point clouds. In particular, the laboratory is experimenting with the use of the CloudWork plugin proprietary of the Leica Cyclone software. The plugin allows you to view the cloud by exactly replicating the settings set on the Cyclone software.

${ }^{4}$ The research is part of a project commissioned by the technical office of the University of Pavia that requires the digital documentation of the entire complex of buildings in the historic headquarters of the central University. Significant activities began in December 2018 which was attended by qualified staff from the DAda-LAB Laboratory together with students from the University of Pavia. The research on the modeling of the complex was concentrated on the courtyard of the Statues and the rooms of the Sala della Crociera of the ancient St. Matteo Hospital, now home to the university archaeological museum on this thematic is undergoing a graduation thesis by the student Fu Hangjun.

\section{References}

Agustín L, Quintilla M (2019) Virtual reconstruction in BIM technology and digital inventories of heritage. Int Arch Photogramm Remote Sens Spatial Inf Sci XLII-2/W15:25-31

Bertocci S, Parrinello S (2007) Rilievo e Piano di Gestione per il Centro storico di Montepulciano. In: Clini P, Lancioni N, Quattrini R (eds) atti del convegno EARCOM 07 Sistemi Informativi per 1'Architettura. Alinea, Firenze, pp 108-113

Calvano M (2019) Disegno digitale esplicito. Rappresentazioni responsive dell' architettura e della città. Aracnee, Roma

Cervellini F (2016) Il disegno come luogo del progetto. Well's Srl, Roma

Colarossi P, Lange J (eds) (1996) Tutte le isole di Pietra. Gangemi, Roma

Dore C, Murphy M (2017) Current state of the art historic building information modelling, Int Arch Photogramm Remote Sens Spatial Inf Sci XLII-2/W5:185-192. https://doi.org/10.5194/isprs-arc hives-XLII-2-W5-185-2017

Jordan-Palomar I, Tzortzopoulos P, García-Valldecabres J, Pellicer E (2018) Protocol to manage heritage-building interventions using heritage building information modelling (HBIM). Sustain MDPI Open Access J 10(4):1-19 
Li D, Wang X, Bai C, and Wu C (2019) Discussion on the problem of regularized reconstruction in HBIM. Int Arch Photogramm Remote Sens Spatial Inf Sci XLII-2/W15:657-662

Lo Turco M (2015) Il Bim e la rappresentazione infografica nel processo edilizio. System Graphic, Roma

Maggi M (2007) Musei alla frontiera. Continuità, divergenza, evoluzione nei territori della cultura, Jaca Book, Milano

Morandotti M et al. (2019) L'università di Pavia, i cortili e gli ambienti monumentali. Un progetto di documentazione digitale e lo sviluppo di sistemi di gestione per la manutenzione programmata. In: Conte A, Guida A (eds) Conference Patrimonio in divenire Conoscere, Valorizzare, Abitare, Reuso VII international conference. Gangemi Editore, Matera. 23-26 Ottobre 2019

Osello A (ed) (2015) Building information modeling geographic information system augmented reality per il facility management. Dario Flaccovio, Palermo

Osello A, Ugliotti FM (eds) (2017) BIM: verso il catasto del futuro. Conoscere, digitalizzare, condividere. Il caso studio della Città di Torino. Gangemi Editore, Roma

Parrinello S (2019) Digital and real: parallel processes of documentation and project. In: Parrinello S (ed) Digital \& documentation. Databases and models for the enhancement of heritage. Pavia University Press, Pavia

Parrinello S, Picchio F, De Marco R, Dell'Amico A (2019) Documenting the cultural heritage routes. The creation of informative models of historical russian churches in upper kama region. Int Arch Photogramm Remote Sens Spatial Inf Sci XLII-2/W15

Pavan A, Mirarchi C, Giani M (2017) BIM: Metodi e strumenti. Progettare, costruire e gestire nell'era digitale. Tecniche nuove, Milan

Pocobelli DP, Boehm J, Bryan P et al (2018) BIM for heritage science: a review. Herit Sci 6:30

Ratti C (2017) La città di domani: come le reti stiano cambiando il futuro urbano. Einaudi, Torino 